\title{
BMJ Open Randomised, open-label, multicentric phase III trial to evaluate the safety and efficacy of palbociclib in combination with endocrine therapy, guided by ESR1 mutation monitoring in oestrogen receptor-positive, HER2-negative metastatic breast cancer patients: study design of PADA-1
}

To cite: Berger F, Marce M, Delaloge $\mathrm{S}$, et al. Randomised, open-label, multicentric phase III trial to evaluate the safety and efficacy of palbociclib in combination with endocrine therapy, guided by ESR1 mutation monitoring in oestrogen receptor-positive, HER2-negative metastatic breast cancer patients: study design of PADA-1. BMJ Open 2022;12:e055821. doi:10.1136/ bmjopen-2021-055821

- Prepublication history and additional supplemental material for this paper are available online. To view these files, please visit the journal online (http://dx.doi.org/10.1136/ bmjopen-2021-055821).

Received 14 August 2021 Accepted 08 February 2022

\section{Check for updates}

(c) Author(s) (or their employer(s)) 2022. Re-use permitted under CC BY-NC. No commercial re-use. See rights and permissions. Published by BMJ.

For numbered affiliations see end of article.

\section{Correspondence to} Dr François-Clement Bidard; francois-clement.bidard@ curie.fr

Frédérique Berger, ${ }^{1}$ Margaux Marce, ${ }^{2}$ Suzette Delaloge, ${ }^{3}$ Anne-Claire Hardy-Bessard, ${ }^{4}$ Thomas Bachelot, ${ }^{5}$ Ivan Bièche, ${ }^{6}$ Anne Pradines, ${ }^{7,8}$ Thibault De La Motte Rouge, ${ }^{9}$ Jean-Luc Canon, ${ }^{10}$ Fabrice André, ${ }^{11}$ Laurent Arnould, ${ }^{12}$ Florian Clatot, ${ }^{13}$ Jérôme Lemonnier, ${ }^{14}$ Sandrine Marques (D) , ${ }^{14}$ François-Clement Bidard, ${ }^{15,16}$ PADA-1 investigators

\section{ABSTRACT}

Introduction The combination of a CDK4/6 inhibitor with an aromatase inhibitor (Al) has recently become the gold standard for Al-sensitive first line treatment of oestrogen receptor-positive (ER+) HER2-negative (HER2-) advanced breast cancer. However, most patients receiving this combination will ultimately progress and require further therapies.

Several studies have demonstrated that the onset of a ESR1 gene mutation lead to Als resistance in the advanced setting. ESR1 mutations can be detected in circulating tumour DNA (ctDNA) using a digital PCR assay. Our study aims to prove the clinical efficacy of periodic monitoring for emerging or rise of ESR1 mutations in ctDNA to trigger an early change from Al plus palbociclib to fulvestrant plus palbociclib treatment while assessing global safety. Methods PADA- 1 is a randomised, open-label, multicentric, phase III trial conducted in patients receiving Al and palbociclib as first line therapy for metastatic ER +HER2- breast cancer. 1000 patients will be included and treated with palbociclib in combination with an Al. Patients will be screened for circulating blood ESR1 mutation detection at regular intervals. Patients for whom a rising circulating ESR1 mutation is detected without tumour progression (up to $\mathrm{N}=200$ ) will be randomised (1:1) between (1) Arm A: no modification of therapy; and (2) Arm B: palbociclib in combination with fulvestrant, a selective ER down-regulator. At tumour progression, an optional crossover will be offered to patients randomised in arm A. The coprimary endpoints are (1) Grade $\geq 3$ haematological toxicities and their associations with baseline characteristics and (2) progression-free survival in randomised patients.

\section{Strengths and limitations of this study}

- PADA-1 is the first-of-its-kind trial to engage the 'subclone war', by targeting ESR1 mutation right after they appear and before disease progression.

- Because of its large size and design, if positive, PADA-1 could be practice changing.

- More potent oral selective oestrogen receptor degraders could yield to greater benefit than fulvestrant.

- Cross-over was optional and postprogression treatments are not standardised, limiting the power of secondary efficacy endpoints that involve postprogression therapies.

- No hypothesis is made to conclude about the toxicity of the palbociclib+fulvestrant combination.

Ethics and dissemination The study has been approved by the French medicines agency (ANSM) and by an ethics committee (ref 01/17_1 CPP Ouest-IV Nantes) in January 2017. The trial results will be published in academic conference presentations and international peer-reviewed journals.

Trial registration numbers EudraCT: 2016-004360-18; NCT03079011.

\section{BACKGROUND}

Significant progresses have been made in detecting and treating breast cancer over the last three decades. Although the relative mortality linked to breast cancer is declining, 
around $20 \%$ of patients with initial early breast cancer will ultimately develop metastases despite adequate adjuvant treatments. Furthermore, $5 \%-7 \%$ of breast cancer patients present with metastases at diagnosis in western countries. Even though survival expectancy is improving, in most cases, breast cancer is considered incurable once it reaches the metastatic stage. ${ }^{1}$ This study investigates the first-line therapy of the most frequent breast cancer subtype oestrogen receptor-positive (ER+) HER2negative (HER2-) metastatic breast cancer (about $70 \%$ of metastatic breast cancer cases).

In patients diagnosed with metastatic or locally advanced ER +HER2- breast cancer not amenable to local curative treatments (surgery or radiotherapy), systemic therapy must be initiated to stop and eventually reduce the tumour burden and increase the patient life expectancy. Costs and treatment-related toxicity are limited in patients receiving endocrine therapy, prompting conferences and cancer guidelines (ABC breast cancer consensus conference; National Cancer Center Network clinical practice guidelines) to recognise endocrine therapy as the gold standard for first-line therapy of metastatic ER +HER2- breast cancer for more than 10 years. ${ }^{23}$

Once tumour progression has been observed under first-line therapy, there are no guidelines for the choice of subsequent lines of treatments. However, some considerations apply: endocrine therapy should be preferred unless chemotherapy seems to be a better optionbecause of poor general status, 'visceral crisis' (that can be defined, according to ABC Consensus, as severe organ dysfunction assessed by signs and symptoms, laboratory studies, and rapid disease progression).

Former trials presented here by chronological order, used the same palbociclib regimen $(125 \mathrm{mg} /$ day orally for 3 weeks followed by 1 week off) and dose adaptation than in PADA-1 trial. In the randomised phase II study PALOMA-1, first-line treatment with the combination of palbociclib plus letrozole achieved a progression-free survival (PFS) of 20.2 months vs 10.2 months with letrozole alone $(\mathrm{p}=0.0004) .{ }^{4}$ Best overall response rate and clinical benefit rate (complete/partial response plus stable disease) for the combination vs letrozole alone were $43 \%$ vs $33 \%$ and $81 \%$ vs $58 \%$, respectively. Subgroup analysis showed that the PFS benefit for the combination was consistent across all subgroups including age, sites of metastatic disease and previous adjuvant therapies. The most common adverse reaction in patients receiving palbociclib plus letrozole was neutropenia. Based on this phase II study, the FDA granted in 2015 accelerated approval to palbociclib for use in combination with aromatase inhibitor (AI) for the treatment of postmenopausal women with HR +HER2- metastatic breast cancer as initial endocrine-based therapy for their metastatic disease. Before granting approval as first line therapy, the EMA chose to wait for the results of the first-line phase III trial, PALOMA-2 (see beyond).

The PALOMA-3 trial assessed the efficacy of palbociclib and fulvestrant in endocrine-resistant advanced breast cancer. ${ }^{5}$ In this study, 521 patients with HR +HER2advanced metastatic breast cancer whose cancer had relapsed or progressed on prior endocrine therapy were randomised to palbociclib and fulvestrant or placebo and fulvestrant. Premenopausal and perimenopausal women were allowed in this study and received Luteinising hormone-releasing hormone agonist. The median PFS was 9.2 months (95\% CI 7.5 to not estimable) with palbociclib-fulvestrant and 3.8 months (95\% CI 3.5 to 5.5) with placebo-fulvestrant (HR 0.42; $95 \%$ CI 0.32 to 0.56 ; $\mathrm{p}<0.001)$. The most common grade 3 or 4 adverse events (AEs) in the palbociclib-fulvestrant group were neutropenia $(62.0 \%$, vs $0.6 \%$ in the placebo-fulvestrant group), leucopenia ( $25.2 \%$ vs $0.6 \%)$, anaemia ( $2.6 \%$ vs $1.7 \%)$, thrombocytopenia $(2.3 \%$ vs $0 \%)$, and fatigue $(2.0 \%$ vs $1.2 \%)$. Febrile neutropenia was reported in only $0.6 \%$ of palbociclib-treated patients and $0.6 \%$ of placebo-treated patients. Based on the PALOMA-3 study, palbociclib was approved in Europe and made available in France for the treatment of advanced, endocrine therapy-resistant HR + HER2- metastatic breast cancer.

In the PALOMA-2 trial, 666 postmenopausal patients with no prior systemic therapy for metastatic breast cancer were randomised 2:1 to receive palbociclib (same schedule than in the above-mentioned studies) combined with an AI (letrozole) or placebo +letrozole until disease progression, consent withdrawal or death. In that study, median PFS was 24.8 months (palbociclib +letrozole) vs 14.5 months (placebo +letrozole) $(\mathrm{HR}=0.58 ; 95 \% \mathrm{CI}$ 0.46 to $0.72 ; \mathrm{p}<0.000001)$. Subgroup analyses regarding the efficacy were negative. Overall response rate was improved with palbociclib +letrozole $(42.1 \%$ vs $34.7 \%$; $\mathrm{p}=0.03 ; 55.3 \%$ vs $44.4 \%$ in patients with measurable disease; $\mathrm{p}=0.01$ ). Clinical benefit rate (including patients with stable disease) was $84.9 \%$ vs $70.3 \%$ ( $p<0.0001) .{ }^{6}$ The first biomarker analysis presented at the 2016 ESMO meeting could not identify predictive markers of palbociclib efficacy. ${ }^{7}$ Common AEs (all grades) observed in the PALOMA-2 study with palbociclib +letrozolevs placebo +letrozole were neutropenia $(79.5 \%$ vs $6.3 \%)$, fatigue $(37.4 \%$ vs $27.5 \%)$, nausea $(35.1 \%$ vs $26.1 \%)$, arthralgia $(33.3 \%$ vs $33.8 \%)$ and alopecia $(32.9 \%$ vs $15.8 \%)$. Grade 4 neutropenia was observed in $10 \%$ of patients. Febrile neutropenia was only seen with palbociclib and letrozole ( $1.8 \%$ of patients). Permanent discontinuation due to AEs was $9.7 \%$ (palbociclib +letrozole) vs $5.9 \%$ (placebo +letrozole). Overall survival data were immature.

Palbociclib is the first-in-class CDK4/6 inhibitor and other inhibitors are being developed. In October 2016, the results of a pivotal phase III trial testing another CDK4/6 inhibitor have been disclosed at the ESMO congress. In the MONALEESA-2 trial, ${ }^{8}$ the addition of ribociclib to letrozole significantly improved PFS in postmenopausal women with ER +advanced breast cancer, with a HR of 0.56 . Median PFS, response rates, and toxicities (except for a possibly higher rate of grade 3/4 liver toxicity in MONALEESA-2) reported inboth study arms were alike 
those observed in the PALOMA-2 trial. Palbociclib can be prescribed in the USA as first-line therapy since the results of PALOMA- 1 and became the standard of care in first line, with more than 40000 US patients treated as of November 2016. In Europe, on 10 November 2016, the European Commission (EC) has approved palbociclib (IBRANCE) in combination with an AI for the treatment of women with HR +HER2- locally advanced or metastatic breast cancer. The approval also covers the use of IBRANCE palbociclib was also approved in combination with fulvestrant for women who have received prior endocrine therapy (as proposed in PADA-1).

ESR1 (oestrogen receptor 1) is the gene coding for the ER $\alpha$ protein. Several studies have shown that ESR1 mutations are rare $(<1 \%)$ at the initial stage of cancer and occurred during the metastatic stage. Approximately 12 ESR1 point mutations have been described, with a hotspot in codons 536-538 in exon 8. These mutations result in a ligand-independent ER activity. In vitro and preclinical data suggest that ESR1 mutations lead to AIs resistance. Schiavon $e t a t^{\theta}$ have developed multiplex droplet digital PCR (ddPCR) assays for ESR1 mutations in circulating tumour DNA (ctDNA) and investigated the clinical relevance and origin of ESR1 mutations in 171 advanced breast cancer women. ESR1 mutation status in ctDNA (see beyond) showed high concordance with contemporaneous tumour biopsies and was accurately assessed in samples shipped at room temperature in preservative tubes. ESR1 mutations were found exclusively in ER+ breast cancer patients previously exposed to AI. Patients with ESR1 mutations had a substantially shorter PFS on subsequent AI-based therapy (HR 3.1; 95\% CI 1.9 to 23.1; $\mathrm{p}=0.004)$. In the SoFEA trial, ESR1 mutations were found in $39.1 \%$ of patients (63 of 161), of whom $49.1 \%$ (27 of $55)$ were polyclonal, with rates of mutation detection unaffected by delays in processing of archived plasma. Patients with ESR1 mutations had improved PFS with fulvestrant $(\mathrm{n}=45)$ compared with exemestane $(\mathrm{n}=18$; HR 0.52 ; $95 \%$ CI 0.30 to $0.92 ; \mathrm{p}=0.02$ ), whereas wild-type ESR1 patients had similar PFS with either treatment (HR 1.07; $95 \%$ CI 0.68 to $1.67 ; \mathrm{p}=0.77) .{ }^{10}$ In the PALOMA-3 trial (advanced metastatic breast cancer, see above), ESR1 mutations were found in the plasma of $25.3 \%$ of patients (91 of 360 ), of whom $28.6 \%$ (26 of 91) were polyclonal, with mutations associated with acquired resistance to prior AI. ${ }^{11}$ Fulvestrant plus palbociclib improved PFS compared with fulvestrant plus placebo in both ESR1 mutant (HR 0.43; 95\% CI 0.25 to $0.74 ; \mathrm{p}=0.002$ ) and ESR1 wild-type patients (HR 0.49 ; $95 \%$ CI 0.35 to 0.70 ; $\mathrm{p}<0.001)$. Therefore, ESR1 mutations can be robustly identified with ctDNA analysis and predict resistance to subsequent AI therapy. Thus, detection of ESR1-activating mutations may be relevant for guiding clinicians between endocrine and non-endocrine therapy.

Tumour cells release fragments of DNA in blood because of their high turnover (ctDNA) and, in cancer patients, ctDNA represents a variable fraction of cell-free DNA (cfDNA). ctDNA differ from normal cfDNA by the presence of cancer-related mutations, as ctDNA fragments harbour the genetic alterations found in the tumour cells of origin. Indeed, several reports showed high concordance between ctDNA mutations, when detectable, and matched tumour mutations. ${ }^{12-14} \mathrm{ctDNA}$ fraction in blood is extremely variable, and can represent from $0.01 \%$ to more than $50 \%$ of cfDNA. ${ }^{15}$

Currently, 'liquid biopsy' approaches depict the mutational landscape of given cancer by qualitative ctDNA analysis; however, the quantitative analysis of ctDNA 'load' in the blood may also be used as a dynamic marker to monitor tumour burden and response to therapy. Several studies reported a correlation between ctDNA changes during therapy with tumour sensitivity to treatment. ctDNA changes may be predictive markers of both responses at the start of treatment and " secondary " resistance during treatment. ${ }^{916-20}$ In addition, two reports showed that rising ctDNA levels can be detected several months before the onset of overt metastatic lesions during follow-up of patients treated for primary breast cancer. The median lead time between rising ctDNA levels and diagnosis of metastatic progression being around 10 months. ${ }^{21} 22$ In the present proposal, we will perform ctDNA analyses using state of the art platforms, with good sensitivity (ddPCR), as part of a collaborative work of the UNICANCER ctDNA group.

In summary, we hypothesised that early change of treatment from AI plus palbociclib to fulvestrant plus palbociclib based on rising ctDNA levels can improve prognosis of patient. To test the clinical utility of this strategy, we proposed a randomised, open-label, multicentric, phase III trial conducted in patients receiving $\mathrm{AI}$ and palbociclib as first-line therapy for metastatic ER+ HER2-negative breast cancer.

\section{METHODS/DESIGN}

This study, including the option of cross-over (not mandatory), has been approved by the French medicines agency (ANSM, Agence Nationale de Sécurité du Médicament et des produits de santé) and by an ethics committee (Comité de Protection des Personnes Ouest IV- Nantes) in January 2017. The overall study design is shown in figure 1.

\section{Aim of the study and endpoints}

The coprimary objectives are (1) to assess whether a change of the endocrine therapy associated with palbociclib (namely, an early switch from AI with palbociclib to fulvestrant with palbociclib following ctDNA detection) will benefit patients in which rising ESR1 mutations are detected during treatment with palbociclib and AI, and (2) to assess the global safety of the combination of palbociclib +endocrine therapy in the whole population of patients, throughout the study, including the potential sequential administration of fulvestrant plus palbociclib, with focus on haematological toxicities. Therefore, coprimary endpoints are (1) PFS measured from the time of 


\section{Palbociclib and ctDNA for ESR1 mutation detection}
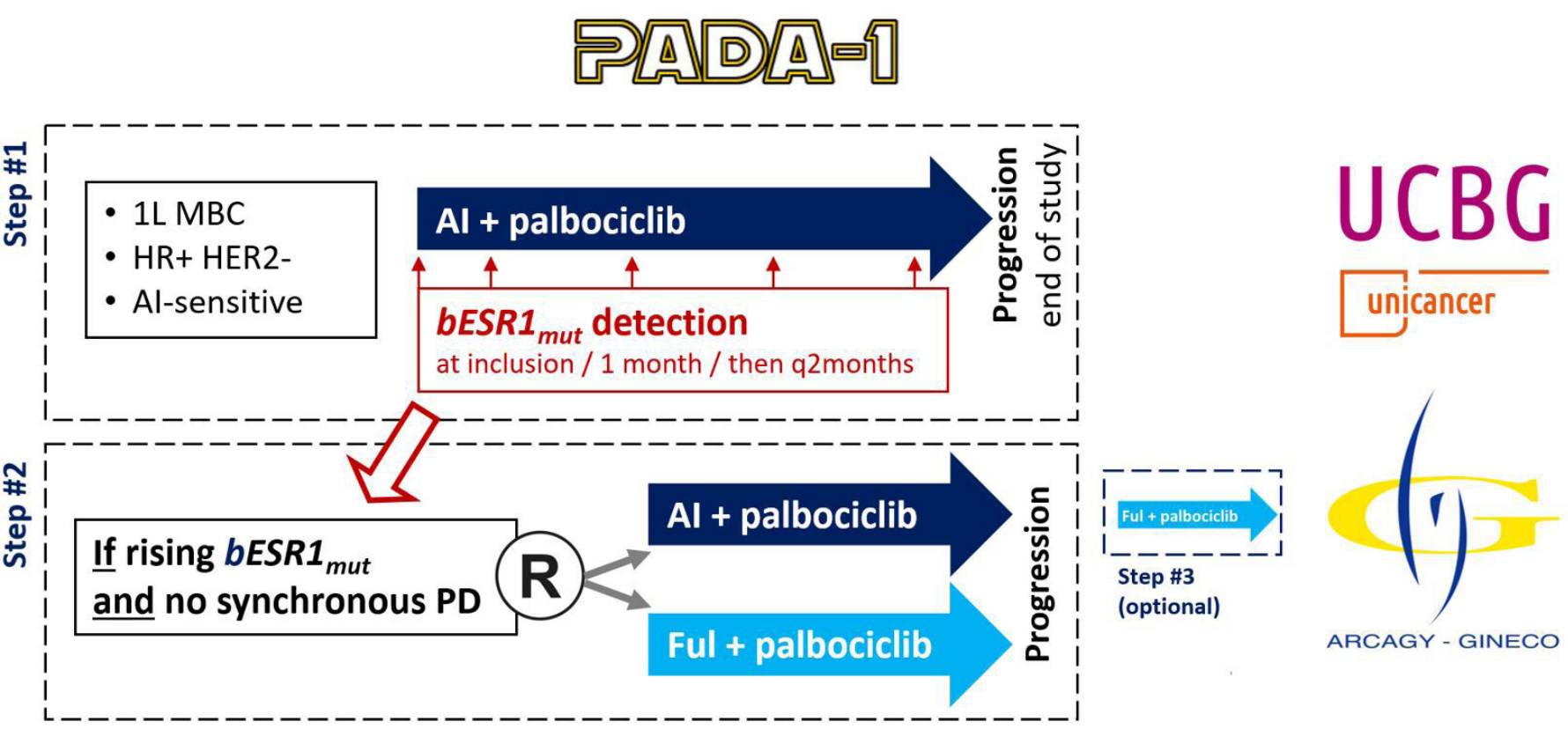

ARCAGY - GINECO

Figure 1 Study scheme. Al, aromatase inhibitor; ctDNA, circulating tumour DNA; PADA-1, Palbociclib and ctDNA for ESR1 mutation detection.

randomisation (following rising ESR1 mutation detection) to the time of tumour progression (as assessed by the investigator per RECIST V.1.1 criteria $^{23}$ ) or death (whichever comes first) - in randomised patients. Patients who have not experienced disease progression and have not died will be censored at the date of last tumour evaluation. Efficacy analyses will be performed using the local radiologist's/investigator's tumour assessments as primary data source. (2) Safety assessed by collection of grade $\geq 3$ AEs, using the Common Terminology Criteria for Adverse Events, V.4.03- in all included patients. The coprimary analysis will focus on grade $\geq 3$ haematological toxicities and their associations with baseline characteristics.

Secondary and translational objectives and endpoints are listed in table 1.

\section{Recruitment}

During the first step, 1000 patients are planned to be included in 83 French centres. Based on the capacities of inclusion from each participating centre, the recruitment phase should last approximately 36 months.

Eligibility criteria are listed in box 1 . After inclusion and non-inclusion criteria have been fulfilled and the patient consent has been obtained, the patient will be included in the step 1 treatment of the trial. Only patients with rising ESR1 mutations will be randomised in the step 2 treatment to either arm A to continue the same therapy (ie, palbociclib-AI combination) or arm B with an early switch to receive palbociclib in association with fulvestrant. Patients will be randomly assigned to one of the two arms (A or $\mathrm{B}$ ) in a 1:1 ratio, using minimisation procedure ${ }^{24}$ stratified according to the presence or not of visceral involvement (defined as any lung, liver, pleura and brain metastasis) and time to rising ESR1 mutation detection ( $\geq 12$ months vs $<12$ months; defined by the time between the date of the first ESR1 mutation detection sampling (baseline evaluation or cycle 1 day 1 visit) and the date of ESR1 mutation detection). The randomisation will be performed using the module of the eCRF/Ennov Clinical software V.7.5.40.7. After the consent form has been signed and all inclusion/non-inclusion criteria checked for step 2, the investigator will proceed with the randomisation through the R\&D Unicancer online electronic case report form. Patients randomised in arm A will be offered to cross-over and be treated by fulvestrant + palbociclib, after disease progression under AI+palbociclib treatment, since Fribbens et al observed an improvement of PFS in patients treated this combination. Palbociclib will be continued as the same dose as before progression (ie, if palbociclib is reduced during step 2 for toxicity, it will not be increased again during step 3). For these patients, the third step (and the trial treatment) will end following tumour progression under fulvestrant + palbociclib.

\section{Trial treatment}

The investigational medicinal products are palbociclib in combination with the standard endocrine therapies, either AI (letrozole, anastrozole or exemestane) or an ER antagonist (fulvestrant). The endocrine therapies and palbociclib should be administered and dose adapted according to Summary of Products Characteristic recommendations and local practice. Palbociclib at a dose of $125 \mathrm{mg}$ will be administered orally at the same time once a day for 21 days followed by 7 days off treatment of every 28-day cycle. Palbociclib capsules will be administered together with AI (letrozole, anastrozole or exemestane) 
Table 1 Secondary and translational objectives and endpoints

\section{Secondary objectives}

\section{Secondary endpoints}

Population included in step 3

To assess PFS in patients undergoing a cross-over following PFS will be measured from the time of cross-over to the time RECIST tumour progression in arm A, from the start of crossover

of tumour progression (as assessed by the investigator per RECIST V.1.1) or death (whichever comes first) - in all patients undergoing the crossover

\section{Population included in step 2 and $3-\operatorname{arm} A$ and $B$}

To assess whether, in patients with rising ESR1 mutations, the early switch (switch following ctDNA detection) to fulvestrant leads a longer time to strategy failure from initial randomisation, than a late switch (cross-over following RECIST tumour progression).

To assess whether, in patients with rising ESR1 mutations, the early switch (switch following ctDNA detection) to fulvestrant leads a longer chemotherapy-free survival from initial randomisation, than a late switch (cross-over following RECIST tumour progression).

All included patients - step 1-3

To report the efficacy (PFS defined on conventional RECIST criteria) of palbociclib combined with hormone therapy (aromatase inhibitor or fulvestrant), from the date of initial inclusion into the trial.

To obtain additional safety data in a broad patient population treated with palbociclib and hormone therapy (aromatase inhibitor or fulvestrant) in a general oncology practice context

To study the patient's reported quality of life before and until 2 years of therapy

Overall survival

\section{Translational objectives (optional)}

To report the quantitative and qualitative analyses of circulating tumour DNA detection before and during therapy, the comparison with archived tumour tissue, clinical/ pathological characteristics and efficacy of therapy.
Time to strategy failure will be measured from the time of randomisation until palbociclib +endocrine therapy discontinuation or death (whichever comes first)

Chemotherapy-free survival will be measured from the time of randomisation until the date of chemotherapy initiation or death (whichever comes first) - in all randomised patients. Anticancer treatments received after the study treatment discontinuation wil be described.

PFS will be measured from the time of inclusion to the time of tumour progression (as assessed by the investigator per RECIST V.1.1) or death (whichever comes first) - in all included patients including those switched to fulvestrant

Description of all extrahaematological grade $\geq 3$ toxicities and SAEs incidence rate in the overall population and each treatment step.

Quality of life score obtained through self-administered QLQ-C30 questionnaire at baseline, at randomisation, and every two cycles until disease progression (including patients who perform a late switch from arm A to B) or until 2 years after inclusion whatever the step if patient did not undergo disease progression or rising ctDNA before 2 years.

Overall survival measured from the date of inclusion to that of the patient's death-in all included patients.

\section{Translational endpoints}

ctDNA detection at different time points

ctDNA, circulating tumour DNA; PFS, progression-free survival; SAE, serious AE.

until randomisation. After randomisation, palbociclib will be given either in association with standard endocrine therapy (letrozole, anastrozole or exemestane) or in association with an ER antagonist (fulvestrant). In the event of significant treatment-related toxicity, palbociclib administration may need to be adjusted. Depending on the nature of the toxicity observed, dosing adjustment may be required for just one or both study drugs in the combination. In the event of significant treatment-related toxicity, palbociclib dosing may be interrupted or delayed and/or reduced as described below. Dose modifications may occur in three ways: (1) Within a cycle: dosing interruption until adequate recovery and dose reduction, if required, during a given treatment cycle; (2) Between cycles: next cycle administration may be delayed due to persisting toxicity when a new cycle is due to start; (3) In the next cycle: dose reduction may be required in a subsequent cycle based on toxicity experienced in the previous cycle. Dose reduction of palbociclib to $100 \mathrm{mg} /$ day, or if needed, to $75 \mathrm{mg}$ /day is recommended depending on the type and severity of toxicity encountered. The use of other concomitant medication/therapy deemed necessary for the care of the patient is allowed.

All medications and therapies taken by the patients or administered to the patients at the onset of trial and all medication given in addition to the IMP during the trial are considered as concomitant medications. The use of other concomitant medication/therapy deemed 


\section{Box 1 Complete list of inclusion and exclusion criteria}

\section{Inclusion criteria (STEP1)}

1. Women with proven locoregionally recurrent or metastatic disease adenocarcinoma of the breast not amenable to curative therapy with disease considered potentially sensitive to aromatase inhibitor. Note: patients relapsing while on adjuvant tamoxifen or other non-aromatase inhibitor adjuvant endocrine therapy and patients relapsing more than 1 year after the end of aromatase inhibitor adjuvant therapy are eligible for this study.

2. Age $\geq 18$ years.

3. Life expectancy $>3$ months.

4. Eastern Cooperative Oncology Group (ECOG) performance status (PS) 0-2.

5. Oestrogen receptor (ER)-positive and HER2-negative breast cancer. Where available, assessment of ER status should be based on the most recent tumour sample; to be considered as ER-positive, the most recent breast cancer tissue examined must display at least $10 \%$ of cancer cells with positive ER staining.

6. Tumour block (primary tumour or metastasis) available.

7. No prior systemic anticancer therapy for metastatic or advanced disease (chemotherapy targeted therapy or endocrine therapy); prior initiation of luteinising hormone-releasing hormone (LHRH) agonist or bone-directed agents is however allowed).

8. Menopausal patients or patients with suppressed ovarian function:

- Women with bilateral oophorectomy

- Postmenopausal women, as defined by any of the following criteria:

- Age 60 or over.

- Age 50-59 years and meets one of the following criteria:

- Amenorrhoea for $\geq 24$ months and follicle-stimulating hormone within the postmenopausal range.

- Patients with hysterectomy or chemotherapy-induced amenorrhoea must display follicle-stimulating hormone within the postmenopausal range.

- Other women, provided they are being treated with monthly LHRH analogues (first injection performed $\geq 7$ days before the treatment initiation) and are willing to continue to receive LHRH agonist therapy for the duration of the trial.

9. Patients may have measurable (according to Response Evaluation Criterion in Solid Tumours (RECIST V.1.1) or not measurable disease:

- Patients with only blastic bone lesions are not eligible.

- Patients with only pleural, cardiac or peritoneal effusion or meningeal carcinomatosis are not eligible.

10. Adequate organ and marrow function as defined below:

- Hqemoglobin $\geq 90 \mathrm{~g} / \mathrm{L}$.

- Absolute neutrophil count $\geq 1.5 \mathrm{G} / \mathrm{L}$.

- Platelet count $\geq 100 \mathrm{G} / \mathrm{L}$.

- Serum bilirubin $\leq 1.5 \times$ ULN (Upper Limit of Normal value). This will not apply to patients with confirmed Gilbert's syndrome.

- ALT (alanine aminotransferase) and AST (aspartate aminotransferase) $\leq 3 \times$ ULN.

- Alkaline phosphatase $\leq 2.5 \times$ ULN.

- Serum creatinine $\leq 1.5 \times$ ULN or calculated creatinine clearance $\geq 60 \mathrm{~mL} / \mathrm{min}$ as determined by Cockcroft-Gault (using actual body weight) formula for females [creatinine clearance=weight $(\mathrm{kg}) \times(140-$ age $) \times 0.85(\mathrm{~mL} / \mathrm{min}) /(72 \times$ serum creatinine $(\mathrm{mg} /$ $\mathrm{dL})$ ).

11. Willingness and ability to comply with scheduled visits, treatment plan, laboratory tests, and any protocol-related procedures including screening evaluations.
Box 1 Continued

12. Resolution of all acute toxic effects or prior anticancer therapy or surgical procedures to $\mathrm{NCl}$-Common Terminology Criteria for Adverse Events V.4.03 grade 1 (except alopecia or other toxicities not considered a safety risk for the patient at investigator's discretion).

13. Written informed consent obtained prior to performing any protocolrelated procedures including screening evaluations.

14. Patient affiliated to a social security system.

\section{Non-inclusion criteria (step 1)}

1. Locally advanced breast cancer or locoregional relapse amenable for any treatment with curative intent.

2. HER2-positive or equivocal tumour status either on the primary or on the recurrent tumour, defined as $\mathrm{IHC}_{+}+$, Fish/Cish amplified or Fish/Cish equivocal according to the ASCO2015 criteria.

3. Prior endocrine therapy in the metastatic setting is not allowed.

4. Prior treatment with any CDK $4 / 6$ inhibitor in the adjuvant or metastatic setting (neoadjuvant/preoperative treatment is allowed); however, prior therapy with another targeted treatment in the adjuvant setting is allowed.

5. Visceral crisis: Advanced, symptomatic, visceral spread that is at risk of life-threatening complication in the short term and that requires chemotherapy.

6. Any major surgery (defined as requiring general anaesthesia) or significant traumatic injury within 4 weeks of treatment initiation or patients that may require major surgery during the course of the study; however, surgical diagnostic procedure is allowed (even if under general anaesthesia).

7. Known active, bleeding diathesis.

8. Any serious known concomitant systemic disorder incompatible with the study (at the discretion of investigator), previous history of bleeding diathesis, or anticoagulation treatment (the use of low molecular weight heparin is allowed).

9. Patients unable to swallow tablets.

10. History of mal-absorption syndrome or other condition that would interfere with enteral absorption.

11. Chronic daily treatment with corticosteroids with a dose of $\geq 10 \mathrm{mg} /$ day methylprednisolone equivalent (excluding inhaled steroids).

12. Known active uncontrolled or symptomatic CNS metastases, carcinomatous meningitis, or leptomeningeal disease as indicated by clinical symptoms, cerebral oedema and/or progressive growth. Patients with a history of CNS metastases or cord compression are eligible if they have been definitively treated with local therapy (eg, radiotherapy, stereotactic surgery) and are clinically stable and off anticonvulsants and steroids for at least 4 weeks before treatment start.

13. Known hypersensitivity to letrozole, anastrozole, exemestane, fulvestrant, palbociclib or any of their excipients.

14. Uncontrolled electrolyte disorders that can compound the effects of a QTC prolonging drug (eg, hypocalcaemia, hypokalaemia, hypomagnesaemia).

15. Patients treated within the last 7 days prior to treatment start in the trial with drug that are known to be CYP3A4 inhibitors, drugs that are known to be CIP3A4 inducers, or with patients who underwent a grapefruit and grapefruit juice cure.

16. Patients already included in another therapeutic trial evaluating an investigational medicinal product or having received an investigational medicinal product within 3 months.

17. History of previous: 


\section{Box 1 Continued}

- Any stage II, III, IV cancer within 5 years preceding patient enrolment in the trial-however, multiple breast cancers (contralateral/ipsilateral cancers/local relapses) are allowed pending all tumour masses were ER+

- Any history of haematological malignancy.

18. Persons deprived of their freedom or under guardianship or incapable of giving consent.

19. Pregnancy or lactation period. Women of childbearing potential must implement adequate non-hormonal contraceptive measures (barrier methods, intrauterine contraceptive devices, sterilisation; LHRH agonist cannot be considered as an efficient contraceptive measure) during study treatment and for 90 days after discontinuation. A serum pregnancy test must be negative in premenopausal women or women with amenorrhoea of less than 12 months.

Inclusion criteria, randomised part (step 2):

1. Patients included and treated within the PADA-1 protocol, who received a combination of aromatase inhibitor and palbociclib.

2. Detection of a rise in circulating ESR1 mutation as defined in the protocol (see part 4.4).

3. Absence of concomitant RECIST V.1.1 proven tumour progression.

4. Life expectancy $>3$ months.

5. ECOG PS 0-2.

6. Patients who have been properly informed and have signed the informed consent of the randomised part of the protocol.

Non-Inclusion criteria, randomised part (step 2):

1. Patients who have stopped the aromatase inhibitor therapy for more than four consecutive weeks.

2. Patients with a visceral crisis linked to their underlying breast cancer.

\section{Inclusion criteria, crossover part (STEP 3):}

1. Patients who have been included in the PADA-1 study, who were randomised for the no-change arm (arm A) on rising ESR1 circulating tumour DNA.

2. Patients who have recent documented tumour progression (RECIST V.1.1).

necessary for the care of the patient is allowed (other than those described below); any medication intended solely for supportive care (eg, analgesics, antidiarrhoeals, antidepressants) may also be used at the investigator's discretion.

\section{Search for circulating ESR1 mutations}

Patients will be access by molecular testing in two regional molecular cancer genetics platforms certified by UNICANCER for the PADA-1 trial.

Mandatory blood samples are required during this study for ESR1 mutations screening in ctDNA. A noncompliance of collection of blood sample for ctDNA measurement will lead to patient withdrawal. For the purpose of the primary objective, blood samples will be collected at inclusion (baseline and/or cycle 1 day 1), after the first cycle (cycle 2 day 1 ), after the third cycle (cycle 4day 1) and then every other two cycles from enrolment in the study until the end of treatment. At each blood collection, $20 \mathrm{~mL}$ of blood will be drawn into
2 Streck tubes. When possible, blood collections will be performed during routine blood tests. Streck blood tubes will be sent by express carrier to the central laboratory at room temperature within 36 hours and should be received within 96 hours of collection. Blood tubes will be first centrifuged at $1500 \mathrm{~g}$ for $10 \mathrm{~min}$ at room temperature to pellet cells. The supernatant ('low-speed' plasma) will then be harvested (and may be stored at $-80^{\circ} \mathrm{C}$ ). In order to extract plasma DNA, 'low-speed' plasma will be centrifuged at $16000 \mathrm{~g}$ for $10 \mathrm{~min}$ at room temperature to remove cell debris. The supernatant ('high-speed' plasma) may be stored at $-80^{\circ} \mathrm{C}$. Cell-free circulating DNA will be extracted from $4 \mathrm{~mL}$ of 'high-speed' plasma using the QIAamp circulating nucleic acid kit (Qiagen) according to the manufacturer's protocol. The extraction procedure can be performed either manually or using automated devices approved by the study biologists. Cell-free circulating DNA may be stored at $-20^{\circ} \mathrm{C}$. Quantification of ESR1 wild type and mutant (ctDNA) alleles will be performed using ddPCR, an amplification-based technique that detect absolute number of molecules. The PCR reaction will be performed using custom-made or commercially available Taqman assays targeting ESR1 exon 5 and 8 mutations, ${ }^{25}$ using the Bio-Rad System. The specificity of each primer pair will be determined on ESR1 DNA in order to define the threshold to discriminate between false and true positive mutant droplets. Each test will contain at least two negative control wells with no DNA and ESR $1_{\text {wwt }}$ DNA as well as an external positive control at $0.5 \%$ ESR1 mutant allele frequency (MAF), which will determine the readability of the test. Results will be expressed by the laboratory as the number of ESR 1 mutant copies detected per $4 \mathrm{~mL}$ of plasma together with the MAF. When the number of mutant ESR1 droplets is null or below the specificity threshold of the assay, the result will be notified 'no detectable ESR1 mutation' to the study sponsor. The turn-around time from sample reception by the laboratory to the results should not exceed a median of 10 working days and cannot exceed 15 working days.

Definition of rising ctDNA: At baseline and/or C1D1 (inclusion in step 1) and first visit (after 1 cycle of treatment for example, C2D1, no patient can be considered displaying a rising ctDNA ESR1 mutation. At the following visits (after C4D1 and then every two cycles), any detectable ESR1 mutation (whatever its level) in patients who had no ESR1 mutation detectable in the previous two blood sampling will be considered as 'rising ctDNA'. An algorithm is predefined by platform geneticist to decide on the 'rising' or 'non-rising' status (online supplemental table 1). In patients with detectable levels of ctDNA ESR1 mutation in at least one of the two most recent analyses, 'rising ctDNA' detection will be defined for each case by the study biologists, considering levels observed at previous time points and the extent of increase. These cases will be discussed by a board including expert oncologists, biologists (representatives of the central genomic platform) and the principal investigator. Note that these 
cases correspond to patients harbouring detectable ESR1 mutation from the very beginning of the study. The number of such patients should be limited, as ESR1 mutation are very uncommon in patients whose cancer is not resistant to AIs.

For translational studies and pending the patient approval (specific consent), all participating patients will be asked to give an additional $20 \mathrm{~mL}$ of blood (drawn on Streck tubes) for further circulating/ctDNA biomarker research during mandatory ESRImut blood assessments at step 1 (baseline, cycle 2 day 1 , cycle 4 day 1 , and progression (if applicable)), step 2 (randomisation, cycle 3 day 1 , cycle 5 day 1 , and progression) and step 3 (cross-over, cycle 3 day 1 , cycle 5 day 1 , and progression). Furthermore, all patients entering the main study will be proposed to participate to the translational tumour sampling study, whose aim to search for genetic changes driving the early or late resistance to the combination of palbociclib and endocrine therapy. This study will be conducted on archived tumour tissue (inclusion) and archived tumour sample (at progression).

\section{Visits and follow-up}

Patients will be monitored from the date of inclusion to the date of disease progression or, for patients randomised in arm A and who cross-over after a first disease progression, until the date of disease progression under fulvestrant +palbociclib (second disease progression). Table 2 summarises the follow-up examination/visit schedule. The study is composed of three steps: (1) Step 1 is from the enrolment of the patient (inclusion visit) until the detection of rising ESR1 mutation, or disease progression; (2) Step 2 is from the randomisation until disease progression and (3) Step 3 is a cross-over (only for patients randomised in the arm A and who progressed). This cross-over offers patients initially under palbociclib + AI the possibility to receive palbociclib +fulvestrant.

Eligible patients with signed informed consent form will have an initial assessment at inclusion including clinical evaluation, disease assessment (preferably by CT scan or MRI), standard blood tests, serum biological markers (CA15.3 and CEA) test, ESR1 mutations in ctDNA detection, quality of life (QoL) questionnaires. Physical examination, biological tests including the two serum markers and ESR1 mutation detection, QoL, and safety assessment will be repeated on the first day of cycle 2 and then every two cycles. At disease progression, patients not randomised or randomised in arm B will stop the study treatment and will be followed during 2 years for their survival status. In Arm A, if considered relevant by the physician, patient will be given the opportunity to perform an optional cross-over to receive palbociclib +fulvestrant until further disease progression. An end of study treatment will occur 30 days after the last day of treatment intake. Following disease progression, the survival status (and the date of death if applicable) and new lines of treatment will be collected every 6 months for 2 years.

\section{Sample size and power calculation}

The study is a randomised, open label, controlled, phase III clinical trial with two coprimary objectives of safety and efficacy.

To assess whether a change of endocrine therapy associated with palbociclib (namely, an early switch from $\mathrm{AI}+$ palbociclib to fulvestrant +palbociclib) will extend the PFS of patients in which rising ESR1 mutations are detected in ctDNA during treatment with palbociclib and AI, we hypothesised: (1) the median PFS of patients whose treatment is unchanged (AI+palbociclib, arm A) will be of 4 months ( $\mathrm{H} 0)$, (2) patients early switching to fulvestrant and palbociclib (Arm B), will display an HR of 0.6 (median PFS of 6.7 months). To highlight such a difference with a power of $80 \%$ and a two-sided alpha risk at 5\%, 180 patients with rising ESR1 mutation must be randomised to observe 120 events (tumour progression). Because of study drop-offs and eventual technical problems, we anticipate including up to $10 \%$ more patients, thus up to 200 patients should be randomised. Based on current scientific reports, we estimate that ESR1 mutations can be detected in the ctDNA of $20 \%$ of patients during AI-based therapy. A population of 1000 patients treated by AI and palbociclib is therefore needed to observe up to 200 patients with rising ESR1. The randomisation will be stratified by site of disease (visceral vs nonvisceral) and the time to rising ESR1 mutation, defined as the time elapsed between inclusion in the study and the detection of rising ESR1 mutations ( $\geq$ to 12 months vs $<$ to 12 months).

To obtain additional safety data in a broad patient population treated with palbociclib +endocrine therapy (AI or fulvestrant) and in a general oncology practice context may help to better understand risk factors for palbociclib-related complications. According to the previous PALOMA-2 study (and confirmed by the MONALEESA-2 study with another CDK4/ 6 inhibitor ${ }^{8}$ ), $10 \%$ of breast cancer patients presented a one or more episodes of grade 4 neutropenia during therapy with CDK4/6 inhibitors (before tumour progression and treatment discontinuation). If patients with bone metastasis at baseline are exhibiting a $15 \%$ rate of grade 4 neutropenia at any time during therapy, this would highlight that this subgroup deserves a particular consideration and/ or surveillance. Assuming that advanced breast cancer patients presenting bone metastases is around 60\%, 600 patients with bone metastasis at baseline (among 1000 metastases breast cancer patients) will allow to highlight $15 \%$ of neutropenia with a precision of $3 \%(95 \%$ CI of $\pm 3 \%)$.

Taken together, with the above-mentioned population of 1000 patients, the study will be able to assess both the efficacy of a change of the endocrine therapy associated with palbociclib with $80 \%$ statistical power, and to report rate of grade 4 neutropenia with a precision of $3 \%$. The two coprimary objectives will be analysed independently. 


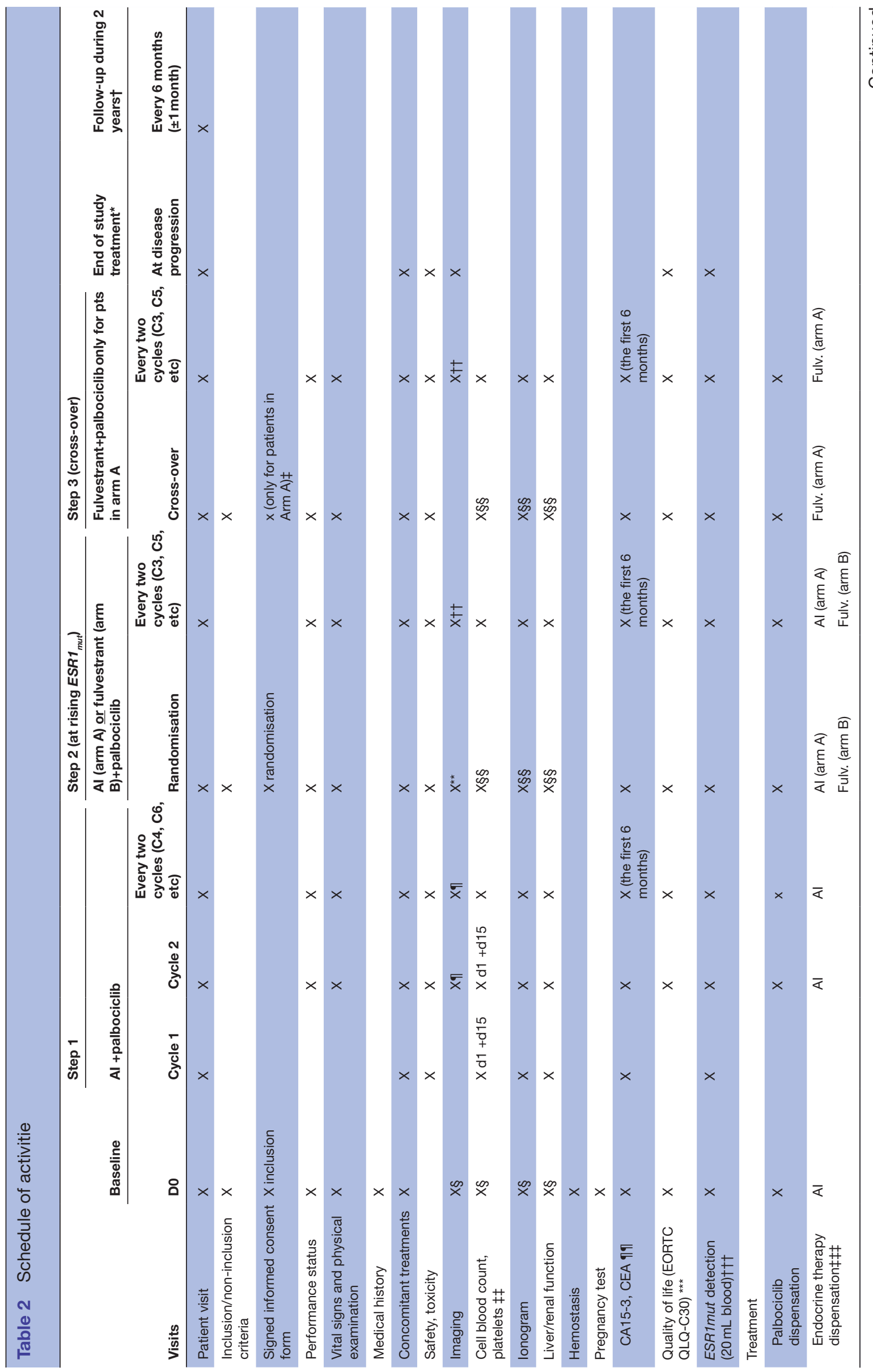

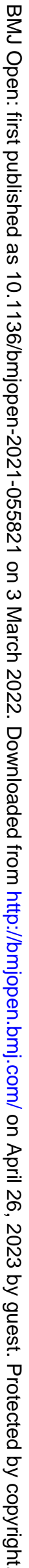




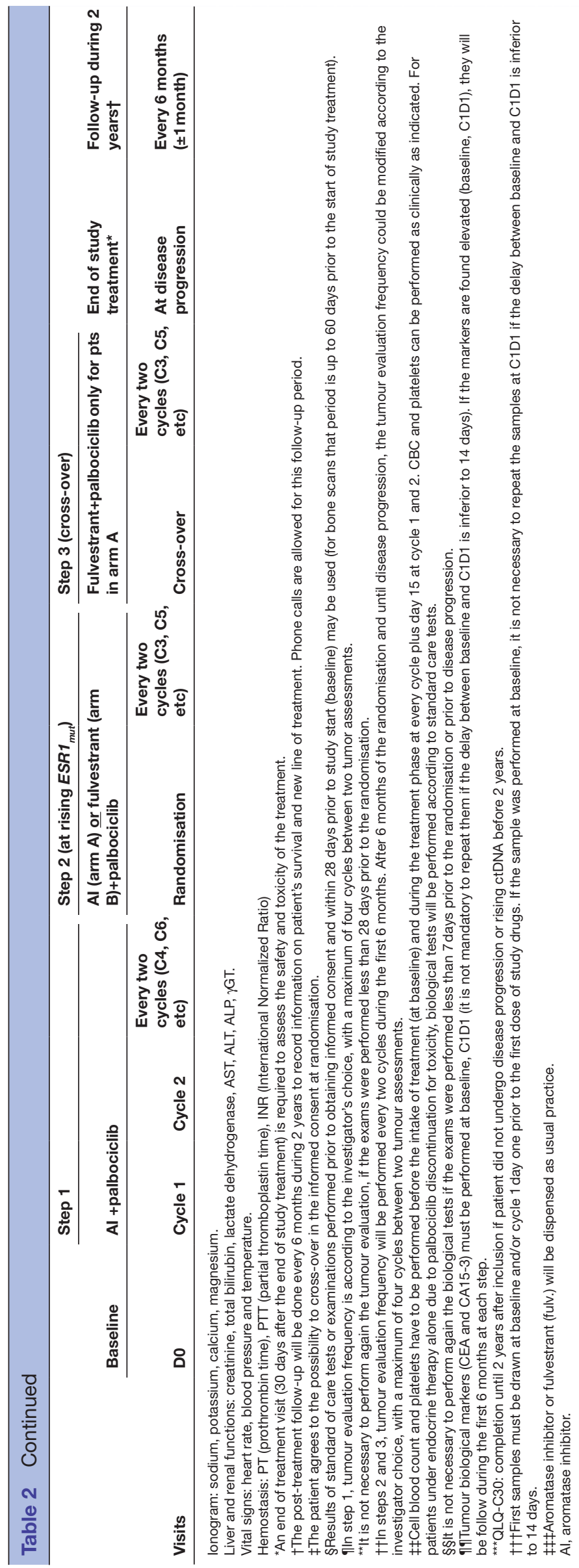

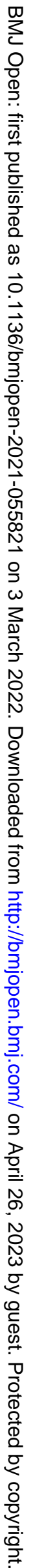


STATISTICAL ANALYSIS

\section{Coprimary outcome}

We propose to test superiority in efficacy analysis using two analysis sets; the 'intention to treat' (ITT) set, considering all patients randomised regardless of whether they received the randomised treatment, and the "per protocol' analysis set, considering all randomised patients who followed the protocol treatment strategy. The superiority of interventional therapy will be declared only if shown to be superior in the ITT analysis set. The distribution of PFS will be estimated for each intervention arm using Kaplan-Meier method and compared using the logrank test. A cox model stratified by site of disease (visceral vs non-visceral) and the time to rising ESR1 mutation will be performed to estimate the treatment HR and its $95 \%$ CI.

Haematological toxicities of grade $\geq 3$ will be summarised by number and percentage with $95 \%$ CI calculated using exact method. The association between incidence of grade $\geq 3$ haematological toxicities and baseline characteristics (Eastern Cooperative Oncology Group Performance Status, metastases localisation, number of metastases site) will be evaluated with $\chi^{2}$ test or Fisher's exact test.

\section{Secondary outcomes}

PFS in all screened patients and in patients proceeded in step 3, time to strategy failure and chemotherapy-free survival will be conducted by the Kaplan-Meier method; HR with two-sided 95\% CIs will be reported. All extrahaematological grade $\geq 3$ toxicities and serious AEs will be summarised by number and percentage in the overall population and each treatment step. The safety profile of palbociclib +endocrine therapy will be analysed considering all patients that received palbociclib. The EORTC-QLQ-C30 questionnaire will be used to report health-related QoL in all included patients. Summary statistics of the scores for all functional/symptom scales will be calculated at each assessment time point.

\section{Translational outcomes}

Number of patients presenting mutations in ctDNA (detection/absence of detection) will be reported with the percentages at each time point. Number of mutant copies and allelic frequencies will be summarised with means, SD, median and range at each time point. Association between ctDNA mutation and clinical factors will be evaluated using $\chi^{2}$ test or Fisher exact test, if appropriate, (for ctDNA mutation as qualitative variable), and Student test or Wilcoxon rank-sum test depending on normality of the data (for ctDNA mutation as continuous variable).

\section{DATA MANAGEMENT}

The management and the monitoring of the data will be realised according to unicancer procedures and is documented in the monitoring plan. Patients will be considered lost to follow-up if no contact can be established despite repeat attempts to contact. Investigators must document all attempts to contact missing patients throughout the study period unless consent for follow-up has been withdrawn. Furthermore, we will report reasons for withdrawal for each randomisation group and compare the reasons qualitatively. Then we expect to have no missing data for the variables required to construct primary and secondary judgement criteria.

\section{DISCUSSION}

Previous research showed that acquired ESR1 mutations in ctDNA are frequently observed after AI exposure in metastatic breast cancer which provide mechanism of resistance to AI therapy. Furthermore, several studies reported the association of ESR1 mutations with poorer PFS and OS. ${ }^{9} 102627$ However, no clinical trial has demonstrated the clinical utility of changing treatment at the onset of the ESR1 mutation by dynamically monitoring ctDNA so far. PADA-1 has been designed to test the clinical utility of an early change of treatment after the rise of ctDNA ESR1 mutations in metastatic breast cancer setting. We expect to detect rising ESR1 mutations in patients treated by AI and palbociclib a few months before the actual radiological tumour progression. A coprimary objective of the study is to test whether an early switch from AI+palbociclib to fulvestrant +palbociclib would increase PFS in patients with rising ctDNA ESR1 mutation without radiological progression. Note that only a fraction of the included patient population will be randomised, as (1) ESR1 mutation is not the only mechanism of resistance and (2) for some patient, ESR1 mutational load may be under the limit of detection of the ctDNA-based screening technology used (we considered that $20 \%$ of patients will have ESR1 mutation detected in ctDNA).

From a methodological point of view, a fair evaluation of the 'early change' of therapy (early switch from AI to fulvestrant before progression), requires a comparison with 'late change' (ie, change after tumour progression). To answer this question, we propose a cross-over for patients with ESR1 mutation detection before tumour progression and randomised to the 'no change' Arm. Assessing PFS under palbociclib plus fulvestrant after tumour progression on palbociclib plus AI will further improved evidence of the clinical utility of ESR1 mutation detection in real time, as performed in the PADA-1 trial.

The second coprimary objective is the global safety of the combination endocrine therapy + palbociblib. While efficacy has been demonstrated, the number of patients in which the palbociclib toxicity profile has been deeply assessed remains limited. Palbociclib is considered as a drug with limited toxicity, yet $10 \%$ of patients experience grade IV neutropenia during treatment. Our study aims at reporting and analysing treatment toxicity with a focus on the only frequent grade IV toxicity, neutropenia (10\% in PALOMA-2 and MONALEESA-2), reported so far with CDK4/6 inhibitors and to analyse this toxicity in regards to the patient baseline characteristics (bone and bone marrow metastasis being eventually involved in that 


\begin{tabular}{ll}
\hline Table 3 Submission history & \\
\hline Submission history & Protocol version \\
\hline Original & Version \#1.0; 14DEC2016 \\
Amendment no 1 & Version \#1.0; 14DEC2016 \\
Amendment no 2 & Version \#1.0; 14DEC2016 \\
Amendment no 3 & Version \#2.0; 07MAY2018 \\
Amendment no 4 & Version \#3.1; 24FEB2020 \\
Amendment no 5 & Version \#4.0; 01OCT2020 \\
Amendment no 6 & Version \#5.0; 24 MAR2021 \\
\hline
\end{tabular}

toxicity). We preferred to use a precision approach to estimate these toxicity rates rather than a statistical test to avoid spending alpha risk on this endpoint, which would have resulted in a significant increase in the number of patients to be included in this trial aiming to validate an innovative therapeutic strategy based on ctDNA and not directly on the drug itself.

\section{Protocol version}

The protocol version number is V.5.0. Submission history is listed in table 3 .

\section{Trial status}

The first patient was enrolled on 22 March 2017. As of 1 July 2021, 1067 patients have registered in step \#1 of the study, 1017 have been included and 172 patients have been randomised in step \#2.

\section{Author affiliations}

${ }^{1}$ Biometry Unit, Institut Curie, PSL Research University, Paris and Saint-Cloud, France

${ }^{2}$ Biometry Unit, Data Center, Institut Régional du Cancer de Montpellier, Montpellier, France

${ }^{3}$ Breast Oncology Department, Gustave Roussy, Villejuif, France

${ }^{4}$ Dapartment of Medical Oncology, Centre Armoricaind'Oncologie, Plérin, France

${ }^{5}$ Department of Medical Oncology, Centre Léon Bérard, Lyon, France

${ }^{6}$ Pharmacogenomic Unit, Genetics laboratory, Department of Diagnostic and Theranostic Medicine, Institut Curie and PSL University, Paris, France ${ }^{7}$ INSERM U1037 CNRS ERL5294 UPS, Cancer Research Center of Toulouse, Toulouse, France

${ }^{8}$ Prospective Biology Unit, Medical Laboratory, Claudius Regaud Institute, Toulouse University Cancer Institute (IUCT-0), Toulouse, France

${ }^{9}$ Department of Medical Oncology, Centre Eugène Marquis, Rennes, France

${ }^{10}$ Department of Medical Oncology, Grand Hôpital de Charleroi, Charleroi, Belgique

${ }^{11}$ Department of Medical Oncology, Gustave Roussy, Villejuif, France

${ }^{12}$ Department of Pathology, Centre Georges François Leclerc, Dijon, France

${ }^{13}$ Department of Medical Oncology, Centre Henri Becquerel, Rouen, France

${ }^{14}$ Research and Development Department, UNICANCER, Paris, France

${ }^{15}$ Department of Medical Oncology, Institut Curie, UVSQ/Paris Saclay University,

Saint Cloud, France

${ }^{16}$ Circulating Tumor Biomarkers laboratory, Inserm CIC-BT 1428, Institut Curie, Paris, France

Collaborators PADA-1 principal investigators: M Achille (Clinique de l'Orangerie, Strasbourg), C Alleaume (Centre Hospitalier Saint-Brieuc, Saint Brieuc), H Ammarguellat (Centre Hospitalier de Beauvais, Beauvais), 0 Arsene (Centre Hospitalier de Blois, Blois), TB (Centre regional Léon Bérard, Lyon), P Barthelemy (Hôpitaux Universitaires de Strasbourg, Strasbourg), S Barthier (Centre Hospitalier de Versailles, Le Chesnay), C Bernard-Marty (Clinique Pasteur, Toulouse), F-CB (Institut Curie - Site Paris, Paris), N Bonichon-Lamichhane (Clinique Tivoli Ducos, Bordeaux), N Bonnin (Centre Hospitalier Lyon Sud, Pierre-Benite), R Bouaita (Centre de radiothérapie et d'oncologie médicale LE-CROME, Ris Orangis), A Boudrant (CH William Morey, Chalon-Sur-Saone), V Brunel (Hôpital Européen Marseille, Marseille), C. Chakiba (Institut Bergonié, Bordeaux), C. Cheneau (Centre Hospitalier Bretagne Sud, Lorient), FC (Centre Henri Becquerel, Rouen), F Dlatot (Institut Claudius Regaud, Toulouse), A De Gramont (Institut Hospitalier Franco-Britannique, Levallois-Perret), T De La Motte Rouge (Centre Eugène Marquis, Rennes), L Deiana (CHRU Morvan, Brest), F Del Piano (Hôpitaux du Léman, Thonon), C Delbaldo (Hopital Diaconesses-Croix Saint Simon, Paris), V Delecroix (Clinique Mutualiste de l'Estuaire, Saint-Nazaire), 0 Derbel (Hôpital privé Jean Mermoz, Lyon), H Desclos (Centre Hospitalier de Broussais, Saint-Malo), V D'Hont (ICM - Val d'Aurelle, Montpellier), N Dohollou (Polyclinique Bordeaux Nord Aquitaine, Bordeaux), C Dubot (Institut Curie - Hôpital René Huguenin, Saint-Cloud), A Escande (Clinique Sainte Anne, Strasbourg), J-M Ferrero (Centre Antoine Lacassagne, Nice), C Foa (Hôpital Saint Joseph, Marseille), J-S Frenel (Institut de Cancérologie de l'Ouest - Site René Gauducheau, Saint-Herblain), M Gardner (Clinique Claude Bernard, Metz), C Garnier Tixidre (Institut Daniel Hollard - Groupe Hospitalier Mutualiste de Grenoble, Grenoble), D Genet (Clinique François Chénieux, Limoges), 0 Gisserot (Clinique du Cap d'Or, La Seine Sur Mer), M Gozy (Clinique de l'Europe, Amiens), C Greilsamer (Centre Hospitalier Départemental de Vendée, La Roche Sur Yon), J Grenier (Institut Sainte Catherine, Avignon), F Guinet (Centre Hospitalier Montceau les mines, Montceau-Les-Mines), A Hardy (CARI0 - Centre Armoricain Radiothérapie Imagerie Médicale et Oncologie, Plerin), J Jacquin (Institut de cancérologie lucien Neuwith, Saint Priest En Jarez), L Joly (Centre Hospitalier René Dubos, Cergy - Pontoise), E Lachaier (CHU Amiens, Amiens), S Ladoire (Centre Georges François Leclerc, Dijon), A-P Laurenty (Clinique de la Croix du Sud, Quint Fonsegrives), R Le Scodan (CHP Saint Grégoire, Saint Gregoire), N Leduc (Centre Catalan d'Oncologie, Perpignan), E Legouffe (Centre ONCOGARD - Institut de Cancérologie du Gard, Nimes), C Levache (Polyclinique Francheville, Perigueux), C Levy (Centre François Baclesse, Caen), F Lorchel (Centre d'Oncologie radiothérapie de Macon, Macon), A Lortholary (Hopital Privé du Confluent, Nantes), B Lucas (Clinique Pasteur-CFR0, Brest), N Marques (Centre Hospitalier Métropole de Savoie, Chambery), A Marti (Centre Hospitalier d'Auxerre, Auxerre), J Medioni (Hopital Européen Georges Pompidou, Paris), A Melis (Centre Hôpital Sainte Marie, Chalon-Sur-Saone), D Mille (Medipole de Savoie, Challes Les Eaux), D Mollon (Centre Hospitalier de Cornouaille, Quimper), L Moreau (Pôle Santé République, Clermont-Ferrand), I Moullet (Clinique de la Sauvegarde, Lyon), M-A Mouret-Reynier (Centre Jean Perrin, Clermont-Ferrand), A Najem (Centre Hospitalier de boulogne sur Mer, Boulogne Sur Mer), S Nguyen (Centre Hospitalier de Pau, Pau), H Orfeuvre (Centre Hospitalier Fleyriat, Bourg En Bresse), J Paitel (CHI Fréjus St-Raphaël, Frejus), T Petit (Centre Paul Strauss, Strasbourg), B Pistilli (Gustave Roussy, Villejuif), J Plaza (UNEOS Site Hopital Robert Schuman, Vantoux), C Riedl (CH Mont de Marsan, Mont De Marsan), R Sabatier (Institut Paoli Calmettes, Marseille), P Soulie (Institut de Cancérologie de l'Ouest - Site Paul Papin, Angers), D Spaeth (Polyclinique de Gentilly, Nancy), L Stefani (Centre Hospitalier Annecy Genevois, Pringy - Metz-Tessy), L Teixeira (Hôpital Saint Louis, Paris), F Trouboul (Centre Hospitalier Bretagne Atlantique, Vannes), C Valmar (Centre Hospitalier le Mans, Le Mans), H Vegas (Centre Hospitalier de Tours - Hopital Bretonneau, Tours), L Venat-Bouvet (CHU Dupuytren, Limoges), A Zannetti (Centre Hospitalier de Cholet, Cholet)

Contributors $\mathrm{FB}$ as principal investigator, $\mathrm{SD}, \mathrm{TB}$ and $\mathrm{A}-\mathrm{CH}-\mathrm{B}$ conceived and designed the study and helped to draft the manuscript. FB contributed to the statistical design of the study, drafted the manuscript and will analyse the clinical data. SD, A-CH-B, TB, IB, AP, TDLMR, FC, J-LC, FA, LA, JL, MM and SM reviewed this manuscript.

Funding The study is funded by Pfizer (grant number not applicable).

Disclaimer Pfizer will not be involved in the collection, analysis, interpretation of the data and writing of scientific manuscripts. Pfizer will have the opportunity to prospectively review any proposed publication, abstract or other type of reports but may not change the conclusions and content.

Competing interests SD: reports grants and non-financial support from Pfizer, grants from Novartis, grants and non-financial support from AstraZeneca, grants and non-financial support from Roche Genentech, grants from Lilly, grants from Puma, grants from Myriad, grants from Orion, grants from Amgen, grants from Sanofi, grants from Genomic Health, grants from GE, grants from Servier, grants from MSD, grants from BMS, grants from Pierre Fabre, outside the submitted work. A-CH-B: reports personal fees from AstraZeneca, personal fees from Daiichi, personal fees from Clovis, personal fees from GSK, personal fees from MSD, personal fees from Novartis, personal fees from Pfizer, personal fees from Roche outside the submitted work. TB: reports personal fees and non-financial support from Roche, grants, personal fees and non-financial support from Novartis, grants, personal fees and non-financial support from AstraZeneca, grants, personal fees 
and non-financial support from Pfizer, personal fees from Seagen, outside the submitted work. TDLMR: reports grants, personal fees and non-financial support from Pfizer, grants and non-financial support from Novartis, personal fees and non-financial support from AstraZeneca, personal fees and non-financial support from Roche Genentech, grants and non-financial support from MSD, personal fees and non-financial support from TESARO-GSK, personal fees from CLOVIS ONCOLOGY, personal fees from MYLAN, outside the submitted work. FA: reports grants from Roche, grants from AstraZeneca, grants from Daiichi Sankyo, grants from Pfizer, grants from Novartis, grants from Lilly, outside the submitted work. LA: report personal fees from Roche, personal fees from MSD, personal fees from AstraZeneca, personal fees fromBMS, outside the submitted work. FC: reports grants from AstraZeneca, grants, personal fees and non-financial support from Roche, personal fees from Lilly, personal fees and non-financial support from Merck Serono, personal fees and non-financial support from BMS, outside the submitted work. F-CB: reports grants from PFIZER, during the conduct of the study; grants, personal fees and non-financial support from PFIZER, grants, personal fees and non-financial support from NOVARTIS, personal fees from LILLY, personal fees and non-financial support from ROCHE, personal fees and non-financial support from AstraZeneca, personal fees from AMGEN, personal fees from SANOFI, personal fees from Radius, grants and personal fees from Seagen, granst from Prolynx outside the submitted work; In addition, F-CB has a patent ctDNA detection by ddPCR pending.

Patient consent for publication Not applicable.

Provenance and peer review Not commissioned; externally peer reviewed.

Supplemental material This content has been supplied by the author(s). It has not been vetted by BMJ Publishing Group Limited (BMJ) and may not have been peer-reviewed. Any opinions or recommendations discussed are solely those of the author(s) and are not endorsed by BMJ. BMJ disclaims all liability and responsibility arising from any reliance placed on the content. Where the content includes any translated material, BMJ does not warrant the accuracy and reliability of the translations (including but not limited to local regulations, clinical guidelines, terminology, drug names and drug dosages), and is not responsible for any error and/or omissions arising from translation and adaptation or otherwise.

Open access This is an open access article distributed in accordance with the Creative Commons Attribution Non Commercial (CC BY-NC 4.0) license, which permits others to distribute, remix, adapt, build upon this work non-commercially, and license their derivative works on different terms, provided the original work is properly cited, appropriate credit is given, any changes made indicated, and the use is non-commercial. See: http://creativecommons.org/licenses/by-nc/4.0/.

ORCID iD

Sandrine Marques http://orcid.org/0000-0002-9101-2589

\section{REFERENCES}

1 Andre F, Slimane K, Bachelot T, et al. Breast cancer with synchronous metastases: trends in survival during a 14-year period. $J$ Clin Oncol 2004;22:3302-8.

2 Cardoso F, Costa A, Norton L, et al. ESO-ESMO 2nd international consensus guidelines for advanced breast cancer (Abc2). The Breast 2014;23:489-502.

3 Cardoso F, Costa A, Senkus E, et al. Correction to: 3rd ESO-ESMO international consensus guidelines for advanced breast cancer (ABC 3). Annals Oncol Off J Eur Soc Med Oncol 2017;28:3111

4 Finn RS, Crown JP, Lang I, et al. The cyclin-dependent kinase 4/6 inhibitor palbociclib in combination with letrozole versus letrozole alone as first-line treatment of oestrogen receptor-positive, HER2-negative, advanced breast cancer (PALOMA-1/TRIO-18): a randomised phase 2 study. Lancet Oncol 2015;16:25-35.

5 Turner NC, Ro J, André F, et al. Palbociclib in hormone-receptorpositive advanced breast cancer. N Engl J Med 2015;373:209-19.

6 Finn RS, Martin M, Rugo HS, et al. PALOMA-2: primary results from a phase III trial of palbociclib $(P)$ with letrozole $(L)$ compared with letrozole alone in postmenopausal women with ER+/HER2advanced breast cancer (ABC). J Clin Oncol 2016;34:507
7 Finn R, Jiang Y, Rugo H. LBA15 - Biomarker analyses from the phase 3 PALOMA-2 trial of palbociclib (P) with letrozole (L) compared with placebo (PLB) plus $L$ in postmenopausal women with ER + /HER2advanced breast cancer (ABC). Abstr Book 41st ESMO Congr ESMO 2016 7-11 Oct 2016. Cph Den 2016;27:vi554.

8 Hortobagyi GN, Stemmer SM, Burris HA, et al. Breast cancer, locally advanced and metastatic first-line ribociclib + letrozole for postmenopausal women with hormone receptor-positive ( $\mathrm{HR+}$ ), HER2-negative (HER2-), advanced breast cancer (ABC). Annals of Oncology 2016;27:vi553.

9 Schiavon G, Hrebien S, Garcia-Murillas I, et al. Analysis of ESR1 mutation in circulating tumor DNA demonstrates evolution during therapy for metastatic breast cancer. Sci Trans/ Med 2015;7:313ra182-313.

10 Fribbens C, O'Leary B, Kilburn L, et al. Plasma ESR1 Mutations and the Treatment of Estrogen Receptor-Positive Advanced Breast Cancer. J Clin Oncol 2016;34:2961-8.

11 Cristofanilli M, Turner NC, Bondarenko I, et al. Fulvestrant plus palbociclib versus fulvestrant plus placebo for treatment of hormonereceptor-positive, HER2-negative metastatic breast cancer that progressed on previous endocrine therapy (PALOMA-3): final analysis of the multicentre, double-blind, phase 3 randomised controlled trial. Lancet Oncol 2016;17:425-39.

12 Douillard J-Y, Ostoros G, Cobo M, et al. Gefitinib treatment in EGFR mutated Caucasian NSCLC: circulating-free tumor DNA as a surrogate for determination of EGFR status. J Thorac Oncol 2014;9:1345-53.

13 Kinugasa H, Nouso K, Miyahara K, et al. Detection of K-ras gene mutation by liquid biopsy in patients with pancreatic cancer. Cancer 2015;121:2271-80.

14 Lebofsky R, Decraene C, Bernard V, et al. Circulating tumor DNA as a non-invasive substitute to metastasis biopsy for tumor genotyping and personalized medicine in a prospective trial across all tumor types. Mol Oncol 2015;9:783-90.

15 Diehl F, Schmidt K, Choti MA, et al. Circulating mutant DNA to assess tumor dynamics. Nat Med 2008;14:985-90.

16 Chang GA, Tadepalli JS, Shao Y, et al. Sensitivity of plasma BRAFmutant and NRASmutant cell-free DNA assays to detect metastatic melanoma in patients with low RECIST scores and nonRECIST disease progression. Mol Oncol 2016;10:157-65.

17 Gray ES, Rizos H, Reid AL, et al. Circulating tumor DNA to monitor treatment response and detect acquired resistance in patients with metastatic melanoma. Oncotarget 2015;6:42008-18.

18 Lipson EJ, Velculescu VE, Pritchard TS, et al. Circulating tumor DNA analysis as a real-time method for monitoring tumor burden in melanoma patients undergoing treatment with immune checkpoint blockade. J Immunother Cancer 2014;2:42

19 Madic J, Kiialainen A, Bidard F-C, et al. Circulating tumor DNA and circulating tumor cells in metastatic triple negative breast cancer patients. Int J Cancer 2015;136:2158-65.

20 Tie J, Kinde I, Wang Y, et al. Circulating tumor DNA as an early marker of therapeutic response in patients with metastatic colorectal cancer. Ann Oncol 2015;26:1715-22.

21 Garcia-Murillas I, Schiavon G, Weigelt B, et al. Mutation tracking in circulating tumor DNA predicts relapse in early breast cancer. Sci Transl Med 2015;7:302ra133-302.

22 Olsson E, Winter C, George A, et al. Serial monitoring of circulating tumor DNA in patients with primary breast cancer for detection of occult metastatic disease. EMBO Mol Med 2015;7:1034-47.

23 Eisenhauer EA, Therasse P, Bogaerts J, et al. New response evaluation criteria in solid tumours: revised RECIST guideline (version 1.1). Eur J Cancer 2009;45:228-47.

24 Pocock SJ, Simon R. Sequential treatment assignment with balancing for prognostic factors in the controlled clinical trial. Biometrics 1975;31:103-15.

25 Jeannot E, Darrigues L, Michel M, et al. A single droplet digital PCR for ESR1 activating mutations detection in plasma. Oncogene 2020;39:2987-95.

26 Spoerke JM, Gendreau S, Walter K, et al. Heterogeneity and clinical significance of ESR1 mutations in ER-positive metastatic breast cancer patients receiving fulvestrant. Nat Commun 2016;7:11579.

27 Clatot F, Perdrix A, Augusto L, et al. Kinetics, prognostic and predictive values of ESR1 circulating mutations in metastatic breast cancer patients progressing on aromatase inhibitor. Oncotarget 2016;7:74448-59. 